\title{
Suppression of hole decoherence in ultrafast photoionization
}

\author{
Antonia Karamatskou $\odot,{ }^{1,2,3}$ R. Esteban Goetz, ${ }^{4,}{ }^{*}$ Christiane P. Koch, ${ }^{4}$ and Robin Santra ${ }^{1,2,3, \dagger}$ \\ ${ }^{1}$ The Hamburg Centre for Ultrafast Imaging, Luruper Chaussee 149, D-22761 Hamburg, Germany \\ ${ }^{2}$ Center for Free-Electron Laser Science, DESY, Notkestraße 85, D-22607 Hamburg, Germany \\ ${ }^{3}$ Department of Physics, Universität Hamburg, Jungiusstraße 9, D-20355 Hamburg, Germany \\ ${ }^{4}$ Theoretische Physik, Universität Kassel, Heinrich-Plett-Straße 40, D-34132 Kassel, Germany
}

(Received 6 February 2020; accepted 11 March 2020; published 13 April 2020)

\begin{abstract}
In simple one-photon ionization, decoherence occurs due to entanglement between ion and photoelectron. Therefore, the preparation of coherent superpositions of electronic eigenstates of the hole in the photoion is extremely difficult. We demonstrate for the xenon atom that the degree of electronic coherence of the photoion in attosecond photoionization can be enhanced if the influence of many-body interactions is properly controlled. A mechanism at low photon energies involving multiphoton ionization is found, suppressing the loss of coherence through ionization into the same photoelectron partial waves. The degree of coherence found between the $4 d_{0}$ and $5 s$ hole states is, on the one hand, limited by Auger decay of the $4 d_{0}$ hole. On the other hand, increasing the population ratio such that a significant portion of the state is in a true superposition of both states renders the maximization of the degree of coherence difficult.
\end{abstract}

DOI: 10.1103/PhysRevA.101.043405

\section{INTRODUCTION}

The preparation and control of wave packets with light has been in the focus of research in chemistry and physics for many decades [1-4]. The precise characterization of excitation and ionization processes plays a crucial role in the quest for control over matter. In particular, the theoretical study of ultrafast hole dynamics and the associated charge migration in molecules has been attracting a lot of attention in the past few years [5-8]. Usually, it is assumed that a coherent wave packet has been prepared in a sudden manner, such that it constitutes a well-defined initial state. However, the question remains how to generate such a coherent state in the first place. The urgency of this question is evident in view of the fact that first measurements have been performed that probe attosecond wave packets $[9,10]$ and hole dynamics following photoionization $[11,12]$.

Experimental attosecond technology has advanced significantly over the past few years, and attosecond pulses can be synthesized in order to obtain shorter pulses in the optical or (X)UV range with variable bandwidths [13-15]. The current record in shortness to the best of our knowledge is a pulse as short as 43 as [16] reaching the water window. This progress as well as the expected pulse shaping possibilities yet to come will open new doors for spectroscopy and applications, which are particularly interesting in view of wave-packet preparation. Using transient-absorption spectroscopy with subfemtosecond pulses [17-19], the dynamics of valence electrons was already investigated on an attosecond timescale.

\footnotetext{
*Present address: Department of Physics, Kansas State University, 116 Cardwell Hall, 1228 North 17th St., Manhattan, KS 66506-2601, USA.

${ }^{\dagger}$ robin.santra@cfel.de
}

In these endeavors, the coherence of the prepared hole states plays an important role. The loss of coherence, or decoherence, in a given quantum system occurs whenever this quantum system becomes entangled with another quantum system (see, e.g., Ref. [20]). In the context of system-bath models, the quantum system of interest is referred to as system and the second quantum system is called bath (or reservoir or environment). In the present problem, hole decoherence is a consequence of entanglement between the photoion ("system") and the photoelectron ("bath"). It was shown that one way to increase the coherence of the ionic wave packet to some extent is to synthesize light pulses as short as possible [18]. Obviously, there is a limit to this approach, and pulse shortness does by no means represent a sufficient condition for a high degree of coherence.

The specific preparation and control of coherent hole wave packets is still challenging because various types of interaction within atoms and molecules lead to decoherence [21]. The question of decoherence for xenon in one-photon attosecond ionization was addressed in Ref. [22] within the time-dependent configuration-interaction singles (TDCIS) approach [23]. TDCIS is particularly well suited for the description of electron-correlation processes [24,25] leading to entanglement between the photoion and the photoelectron. Reference [22] identified broadband attosecond pulses spanning the binding energies of the xenon $4 d_{0}$ and $5 \mathrm{~s}$ orbitals to be merely a necessary condition for achieving coherence in photoionization. Because an electron from either orbital can be promoted to the same final continuum state $\varepsilon p$ by one-photon ionization, it leaves behind the ionic hole in a partially coherent superposition of a $4 d_{0}$ and $5 s$ hole state. However, since the probability to ionize a $4 d$ electron into an $\varepsilon f$ state is larger than into an $\varepsilon p$ state, the part of the hole state being in a superposition of a $4 d_{0}$ and $5 s$ hole is small. 
The interaction of the ionized electron with the ionic hole during the ionization process results in additional entanglement of the two, further reducing the coherence within the photoion. This effect can —in that simple approach—only be remedied by increasing the mean photon energy of the pulse into the far XUV [22] in order to avoid long interaction times and, therefore, entanglement between the photoelectron and the photoion. However, a high degree of coherence is not guaranteed because of the various accessible photoelectron final states.

In this work, we report on an approach to meet the challenge of preparing coherent wave packets in many-body systems upon attosecond photoionization. It involves, in addition to one-photon processes, three-photon ionization. We demonstrate that the degree of coherence (DOC) can be maximized by controlling the impact of many-body correlations, not necessarily through a shorter interaction time, but using multiphoton ionization, which leads to the same partial waves for the photoelectron while leaving behind a coherent superposition of the hole, and nontrivial chirps. In strong contrast to Ref. [22], in the present work we account for all possible ionization paths, in particular the possible photoelectron $f$ states, and the Auger lifetime of the $4 d_{0}$ hole, in order to model real experimental conditions.

\section{THEORETICAL FRAMEWORK}

In order to learn how coherent wave-packet preparation can be achieved in a true many-body system, we apply quantum optimal control theory (QOCT). It has been employed to tackle the question of control over matter with electromagnetic fields [26,27]. For weak fields, bichromatic control, i.e., the pathway interference between transitions driven by two different photon energies, was discussed in the context of quantum control theory by Shapiro and Brumer [28]. By combining QOCT [27] with the many-body TDCIS method for calculating photoelectron spectra $[29,30]$, quantum interferences among eigenstates involved in the ionization dynamics were exploited specifically for steering photoelectrons [31] and investigating ionic hole coherence in argon [32].

We apply the optimization method of Ref. [32] to photoionization from the xenon $4 d_{0}$ and $5 s$ orbitals to maximize the degree of hole coherence,

$$
g_{i, j}(t)=\frac{\left|\rho_{i, j}^{\mathrm{IDM}}(t)\right|}{\sqrt{\rho_{i, i}^{\mathrm{IDM}}(t) \rho_{j, j}^{\mathrm{IDM}}(t)}},
$$

which is given by the coherence between the hole states $i$ and $j$ in terms of the ion density matrix element, $\rho_{i, j}^{\mathrm{IDM}}$, normalized with respect to the corresponding populations. The simple control mechanism found for argon, of ionizing the $3 p_{0}$ orbital and refilling it by excitation from the $3 s$ orbital [32], fails in this case since the $4 d_{0}$ and $5 s$ orbitals cannot be coupled by one-photon excitation. Given the Auger lifetime of 2.6 fs for the $4 d_{0}$ hole, we restrict our analysis to attosecond photoionization and constrain the pulse duration to a fewhundred attoseconds. In order to avoid the "trivial" solution of reducing the interaction time, and thus entanglement, between photoelectron and ionic hole by increasing the photon energy like in Ref. [22], we limit the photon energies to below $\omega_{\max }=130 \mathrm{eV}$. We ensure the validity of the TDCIS
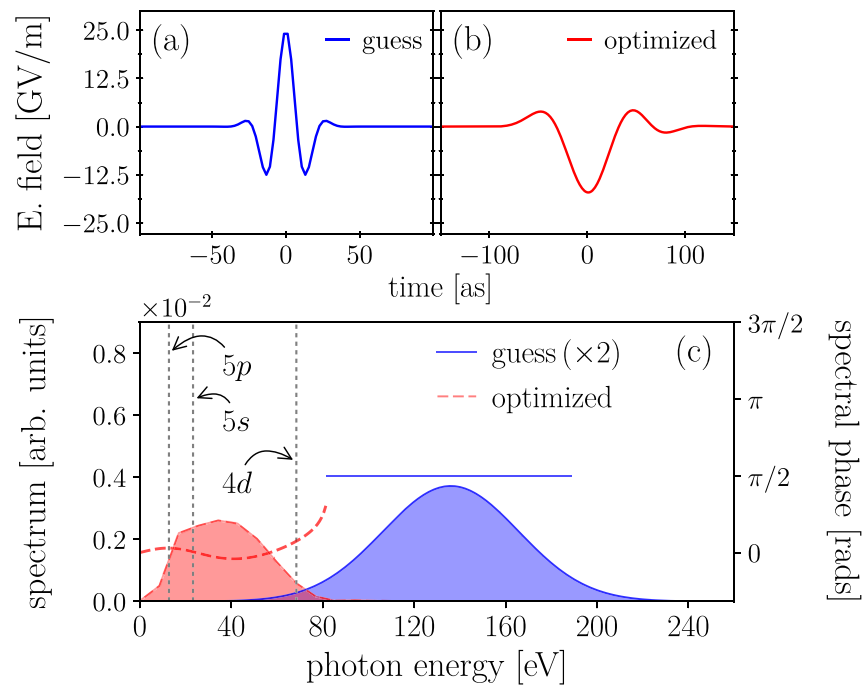

FIG. 1. (a) Gaussian guess field of 20 as pulse duration centered at $136 \mathrm{eV}$ (cf. Ref. [22]), (b) optimized pulse, and (c) power spectra and spectral phases for both pulses.

approximation by considering electric-field amplitudes up to $52 \mathrm{GV} / \mathrm{m}$ for which the probability for multiple ionization remains small. In particular, we choose the amplitude such that the final ground-state depletion is between 0.2 and 0.3 . The upper bound ensures ionization of predominantly a single electron; the lower one ensures reasonably high ionization probabilities for the purpose of experimental detection of photoion coherence.

\section{RESULTS}

The optimized pulse is shown in Fig. 1 together with the guess pulse, which was chosen to be the pulse used in Ref. [22]. Note that the duration of the optimized pulse is much longer than for the pulses considered in Ref. [22]. As shown in Fig. 1(c), the main photon energies of the optimized pulse are redshifted compared to the spectrum of the guess pulse. This is in striking contrast to the control strategy of fast escape by increasing the photoelectron's kinetic energy [22], which would lead to a large blueshift. The main peak of the optimized pulse is centered below the $4 d$ binding energy. Thus, one-photon ionization of the $4 d$ orbital will not be dominant, and the optimized pulse produces slower photoelectrons from ionization of the valence shells than the guess pulse. Figure 1(c) also shows the spectral phases of the pulses. Whereas the Gaussian guess pulse comes with a flat spectral phase, the optimized pulse exhibits nonlinear chirps in the relevant spectral region up to $75 \mathrm{eV}$. By fitting to a polynomial, the spectral phase is found to be approximately cubic. This indicates that in addition to the spectral amplitudes at specific energies, interferences are important for the observed control. Note that it is known that chirps can be used to focus or broaden wave packets [33].

Figure 2 shows the hole populations and the DOC as a function of time for the two pulses. Compared to the dynamics driven by the guess pulse, the optimized pulse increases the ionization of the $5 \mathrm{~s}$ orbital, while decreasing that of the $4 d_{0}$ orbital. While the guess pulse leads to almost no coherence 

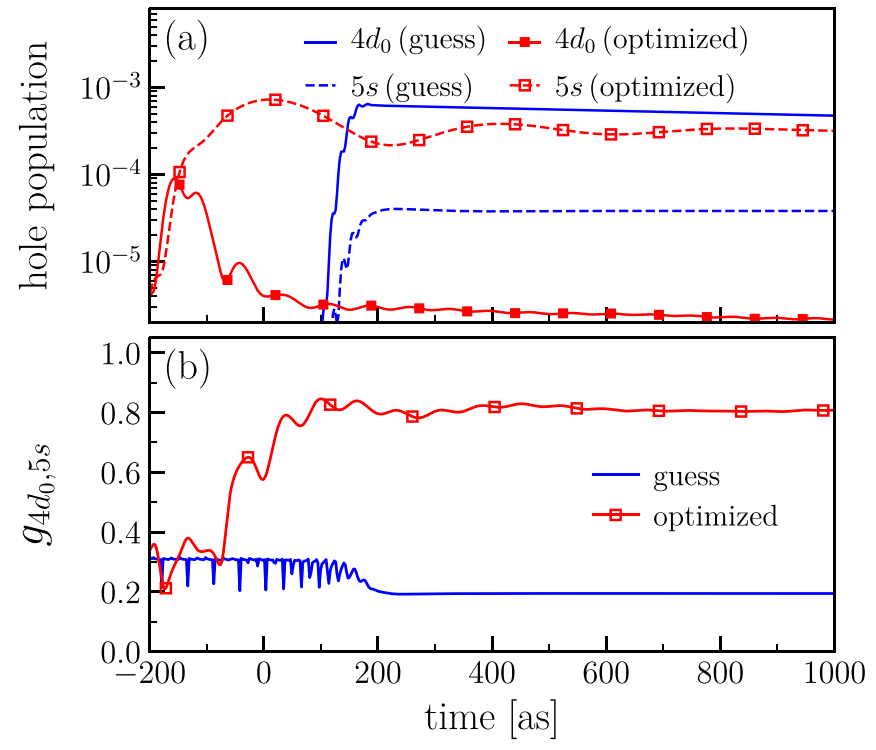

FIG. 2. (a) Hole populations of the $4 d_{0}$ (solid curves) and $5 \mathrm{~s}$ (dashed curves) orbitals resulting from the guess pulse (blue curves) and the optimized pulse (red curves). (b) DOC as a function of time.

at all, the optimized pulse allows the system to reach a value of about 0.85 at the end of the pulse. It prepares the hole in such a superposition that long after the pulse is over, the DOC remains almost constant. However, this comes at the expense of a small hole population in the $4 d_{0}$ orbital, which makes it possible to suppress the impact of Auger decay. In fact, manually switching off the Auger decay in the calculations leads to an almost perfect DOC, $g_{4 d_{0}, 5 s} \approx 1$, compared to 0.85 in Fig. 2(b).

The resulting partial-wave-resolved, angle-integrated photoelectron spectra (PES) for the relevant orbitals are compared in Fig. 3. The partial-wave-resolved PES resulting from the guess pulse and the optimized pulse show significant differences. For the guess pulse, the $4 d_{0}$ ionization dominates, while $5 s$ electrons are ionized much less. The $5 s$ electrons are promoted to an $l=1$ state, whereas the spectrum for the $4 d_{0}$ electrons consists of two parts. At low energies, a final $p$ state is assumed, while at higher energies, an angular momentum of $l=3$ dominates. In total, the amount of ionization into $l=3$ states is much larger than into $l=1$, which is also expected from the Clebsch-Gordan weights (note the logarithmic scale).

In contrast, the optimized pulse produces a strongly modified PES. The photoelectron energies between 5 and $12 \mathrm{eV}$ resulting mainly from $4 d$ ionization are suppressed and the main photoelectron peaks of both $4 d_{0}$ and $5 s$ electrons are observed at very low kinetic energies around $0.1-0.5 \mathrm{eV}$. Intuitively, we can understand this tendency of the optimization as follows: in the range between 75 and $140 \mathrm{eV}$ photon energy [cf. Fig. 1(b)], strong electron correlations occur [24,34] which generate a pronounced response of the $4 d$-shell electrons. By decreasing the mean photon energy of the pulse below the giant resonance, the influence of the strong many-body response is diminished. Generally, the shapes of the partial spectra of the $4 d_{0}$ and the $5 s$ orbitals for the same $l$ states coincide nicely over the whole energy range. The part of

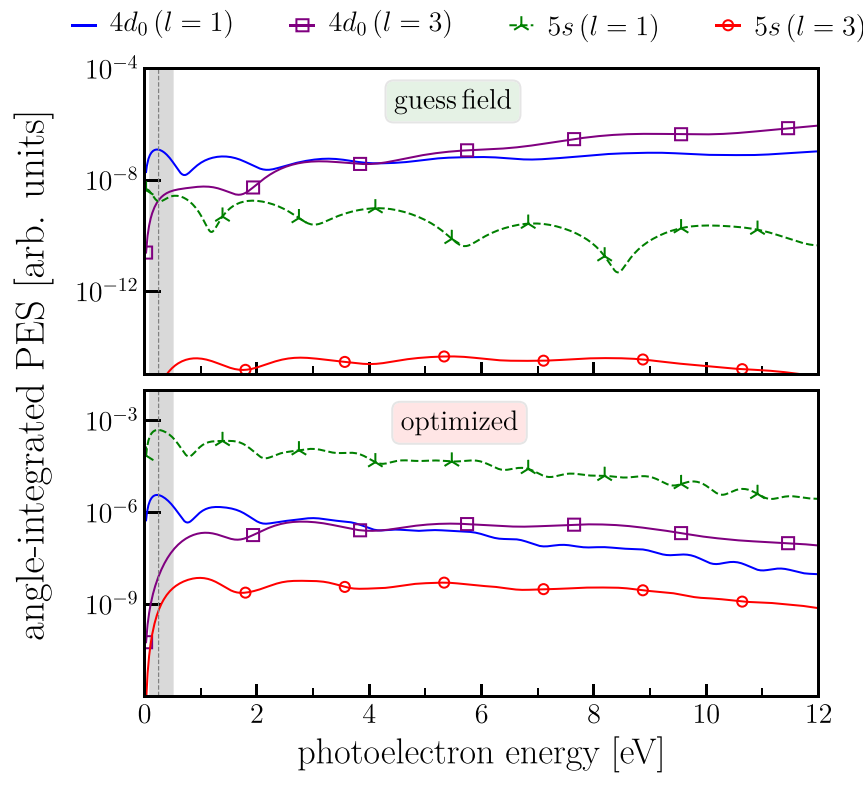

FIG. 3. Partial-wave-resolved, angle-integrated PES for the guess and optimized pulse. Note the peaks below $1 \mathrm{eV}$ whose shape coincides for the corresponding $4 d_{0}$ and $5 s$ ionization.

the final state that is in a superposition of the $5 s$ and $4 d_{0}$ holes with photoelectron angular momentum $l=1$ is strongly enhanced (as compared to $l=3$ at larger kinetic energies) and dominates in particular at low energies.

To scrutinize the impact of many-body correlations and to test the advantageous influence of multiphoton processes, the control strategy is constrained to photon energies below $65 \mathrm{eV}$ in order to exclude one-photon ionization of the $4 d$ shell. At the same time, with the inherently multichannel, many-body approach of TDCIS, we study the impact of electron-correlation effects on the success of the optimization by manually switching correlations on (correlated model) and off (reduced), and comparing the results.

As shown in Fig. 4(b), both optimizations lead to pulse spectra that are strongly peaked at an energy of about $23 \mathrm{eV}$, which can ionize the $5 s$ orbital with one photon and the $4 d_{0}$ orbital via three-photon ionization. Consequently, these pathways can interfere by leading to the same final photoelectron $p$ state with a kinetic energy of about $0.3 \mathrm{eV}$, as shown in Fig. 4(b) in the angle-integrated PES. Therefore, a coherent superposition in the photoion can be created, which leads to a high degree of hole coherence. In Fig. 4(c), the DOCs are shown for both cases: A DOC of 0.88 is obtained for the full, correlated optimization, limited solely by Auger decay. A slightly lower DOC is obtained in the reduced model.

The shapes of the photoelectron spectra shown in Fig. 4(d) are similar for both, the correlated and the reduced scheme. While in the scenario including all correlations the field can strongly drive atomic resonances and change the hole states through channel interactions, in the case without electron correlations the hole state cannot be changed once created. Although one might expect that many-body correlations are hindering the creation of a high DOC, we find in the energyconstrained case that the optimization succeeds in producing significantly enhanced DOCs in both scenarios [cf. Fig. 4(c)], 

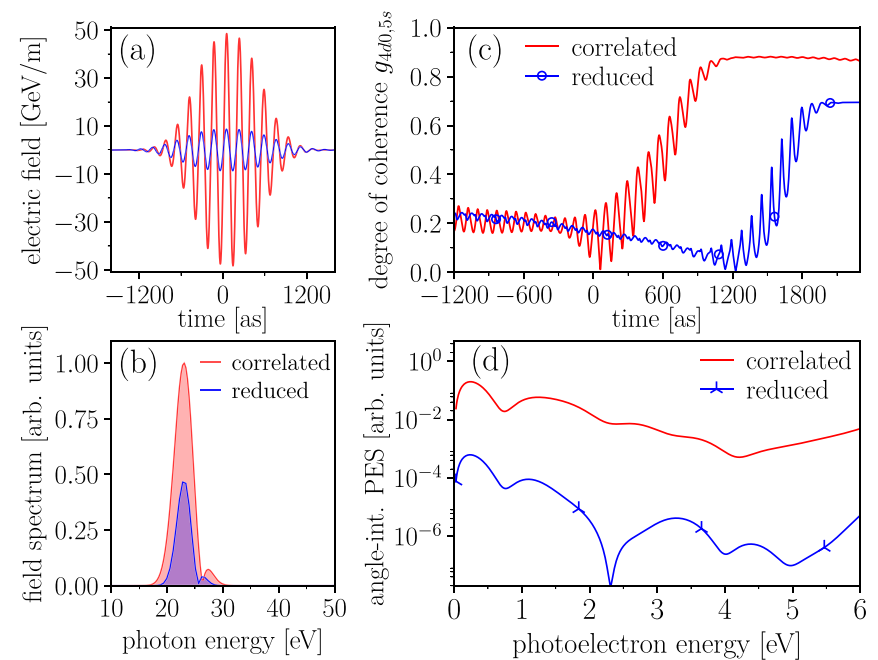

FIG. 4. (a) Energy-constrained optimized fields for the correlated (red) and reduced (blue) models, respectively. (b) Spectra of the corresponding energy-constrained fields. (c) DOC for the correlated and reduced models. (d) Angle-integrated PES for the correlated and reduced-optimized cases.

with the DOC of the correlated model even exceeding the reduced one.

To confirm that the underlying mechanism responsible for the strongly enhanced coherence is indeed interfering multiphoton ionization pathways, the $4 d$ and $5 s$ yields are studied as a function of the peak intensity $\mathcal{I}_{0}$ for the field shape shown in Fig. 4(a) (red curve). The results are shown in Fig. 5. From leading-order perturbation theory, one would expect the $5 s$ yield to scale linearly with the intensity and a power-of-three law for the $4 d_{0}$ yield, which would unambiguously corroborate the hypothesis of coherent wavepacket interference originating from single- and three-photon ionization pathways. From Figs. 5(a) and 5(b), however, it is apparent that the atomic response cannot be described perturbatively for intensities at which a high DOC is achieved [cf. Fig. 5(c)]. At such intensities, the atomic response already starts to saturate. This explains the scaling of the $5 s$ hole population with a power law less than one [Fig. 5(b)]. For the same reason, the power law associated with the intensity

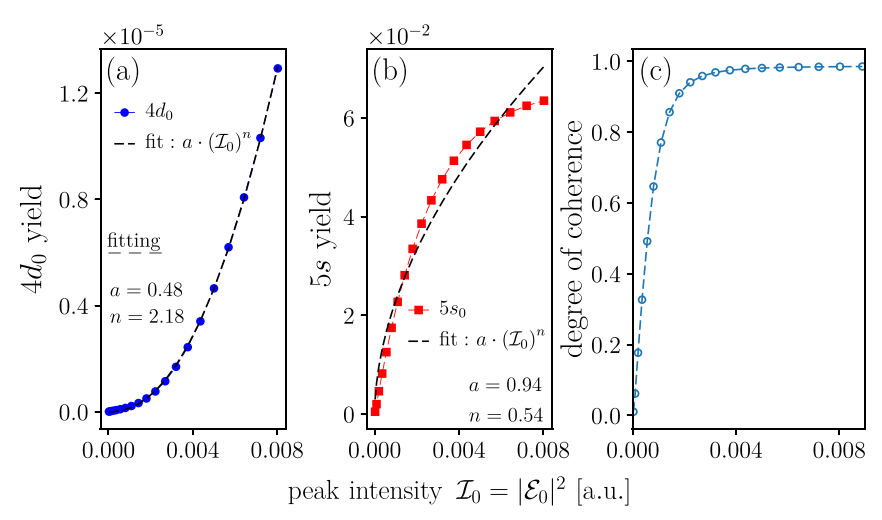

FIG. 5. (a) $4 d_{0}$ and (b) $5 s$ yields as a function of peak intensity; (c) corresponding DOC.

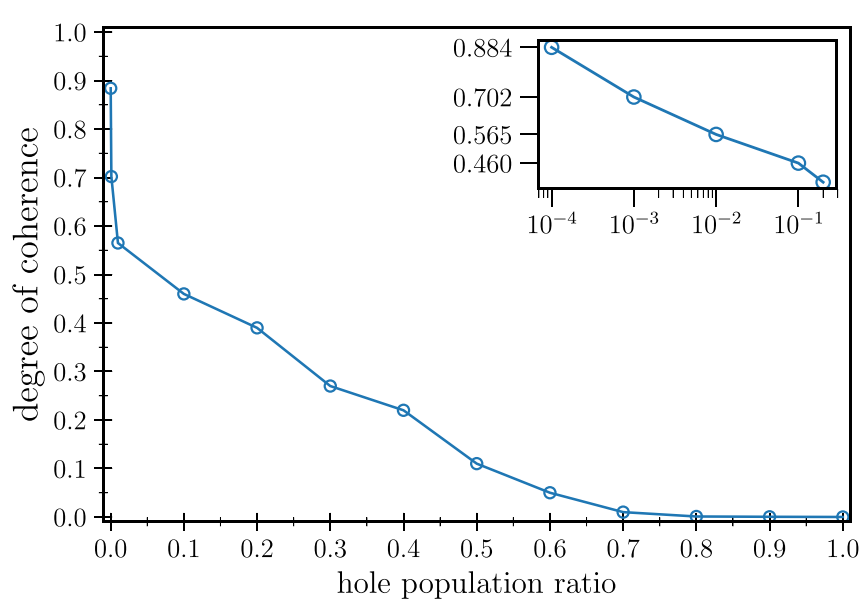

FIG. 6. Maximum achievable degree of coherence for several hole population ratios, $\rho_{4 d_{0}, 4 d_{0}}^{\mathrm{IDM}} / \rho_{5 s, 5 s}^{\mathrm{IDM}}$.

dependence of the $4 d_{0}$ hole population is less than three. But since that power exceeds two-in a regime of (partial) saturation-it is clear that the process in question is (at least) a three-photon process.

Another crucial ingredient in creating a truly coherent superposition of two distinct states is that both states are sufficiently populated with a population ratio of one, or close to one.

In this context, our calculations show that optimizing the DOC while also optimizing the hole population ratio to one dramatically alters the maximum achievable DOC. While independent optimization of either the population ratio or the DOC leads to a high value of the respective quantity, we found that simultaneous optimization of both quantities is rather hard to achieve. In fact, optimization of the DOC with the population ratio as a constraint (in addition to those with respect to the field and the depletion of the ground state) is found to be inefficient as the overall functional is very sensitive to the optimization weights associated with the DOC and the population ratio. In particular, if the optimization weights are comparable, the algorithm tends to arbitrarily favor either the DOC or the population ratio. To overcome this, two strategies were adopted. In the first approach, the algorithm is rendered monotonic by allowing an update of the field only if the ratio and the DOC are simultaneously improved. In the second approach, the hole population ratio is first optimized and, as a second step, the resulting optimized field serves as a guess field for optimization of the DOC, which gives more flexibility for the choice of optimization weights between the DOC and the population ratio in the second step. Both methods render the field update extremely slow, but, more importantly, both give the same overall result, indicating the detrimental effect of large population ratios on the DOC.

In order to find the best compromise between the DOC and the hole population ratio, we proceed to optimize the DOC for fixed hole population ratios. The optimization results are shown in Fig. 6. For every hole population ratio shown on the $x$ axis, the $y$ axis indicates the maximum achievable DOC for that particular ratio. For small ratios, a high DOC can be achieved. For comparable hole populations, the DOC 
decreases very fast. The decreasing value of the DOC as a function of the population ratio clearly indicates the limitations of achieving a high DOC for similar hole populations.

\section{CONCLUSIONS}

In summary, we have explored a mechanism that exploits multiphoton ionization and leads to a noticeable enhancement of the DOC at low photoelectron kinetic energies. By constraining the photon energies and bandwidth of the engineered pulses, the interferometric multiphoton mechanism presented in this work is-by construction - in contrast to that discussed in Ref. [22], where the mean photon energy had to be increased to achieve a high DOC. Here, the DOC can be effectively increased through quantum interference between the predominant single and multiphoton ionization of $5 s$ and $4 d_{0}$ electrons into the same final electronic continuum state with $l=1$ character at low photoelectron energies, and through the simultaneous suppression of ionization into $f$ states. Although the freed electron can still interact with the ionic system at the resulting slow photoelectron kinetic energies, a significant part of the final state can be prepared in a coherent superposition of the $4 d_{0}$ and $5 s$ hole states when introducing appropriate chirps in the pulse. However, preparing the final state in a true superposition of both the $4 d_{0}$ and the $5 s$ states by imposing comparable and stationary hole population ratios after the pulse is over adds an additional constraint to the optimization problem-in addition to the imposed maximum electric-field amplitude, photon energies below $65 \mathrm{eV}$, maximum pulse duration (limited by the Auger decay of the $4 d_{0}$ ), and final ground-state depletion considered in this work-resulting in a decrease in the maximally achievable DOC, compared to the case of unconstrained hole population ratio. Therefore, in the context of engineering pulses under such constraints, a compromise between the two quantities must be found.

In view of the rapid developments of measurement techniques in attosecond physics (see, e.g., Refs. $[14,18]$ ) that make it possible to probe the ionic hole state on an attosecond timescale, the experimental realization of the proposed mechanism for the control of coherent electronic states in atoms and molecules seems feasible in the near future.

\section{ACKNOWLEDGMENTS}

This work was funded by the Cluster of Excellence "Advanced Imaging of Matter" of the Deutsche Forschungsgemeinschaft (EXC 2056, Project ID No. 390715994) and by the State Hessen Initiative for the Development of Scientific and Economic Excellence (LOEWE) within the focus project ELCH.
[1] T. C. Weinacht, J. Ahn, and P. H. Bucksbaum, Nature (London) 397, 233 (1999).

[2] R. Kienberger, M. Hentschel, M. Uiberacker, C. Spielmann, M. Kitzler, A. Scrinzi, M. Wieland, T. Westerwalbesloh, U. Kleineberg, U. Heinzmann et al., Science 297, 1144 (2002).

[3] A. Borot, A. Malvache, X. Chen, A. Jullien, J. Geindre, P. Audebert, G. Mourou, F. Queré, and R. Lopez-Martens, Nat. Phys. 8, 416 (2012).

[4] D. Brinks, F. D. Stefani, F. Kulzer, R. Hildner, T. H. Taminiau, Y. Avlasevich, K. Müllen, and N. F. van Hulst, Nature (London) 465, 905 (2010).

[5] L. Cederbaum and J. Zobeley, Chem. Phys. Lett. 307, 205 (1999).

[6] J. Breidbach and L. S. Cederbaum, Phys. Rev. Lett. 94, 033901 (2005).

[7] F. Remacle and R. D. Levine, Proc. Natl. Acad. Sci. 103, 6793 (2006).

[8] A. I. Kuleff and L. S. Cederbaum, Phys. Rev. Lett. 98, 083201 (2007).

[9] S. Haessler, J. Caillat, W. Boutu, C. Giovanetti-Teixeira, T. Ruchon, T. Auguste, Z. Diveki, P. Breger, A. Maquet, B. Carré, R. Taïeb, and P. Salières, Nat. Phys. 6, 200 (2010).

[10] G. Sansone, F. Kelkensberg, J. F. Pérez-Torres, F. Morales, M. F. Kling, W. Siu, O. Ghafur, P. Johnsson, M. Swoboda, E. Benedetti et al., Nature (London) 465, 763 (2010).

[11] F. Calegari, D. Ayuso, A. Trabattoni, L. Belshaw, S. De Camillis, S. Anumula, F. Frassetto, L. Poletto, A. Palacios, P. Decleva et al., Science 346, 336 (2014).

[12] P. M. Kraus, B. Mignolet, D. Baykusheva, A. Rupenyan, L. Horný, E. F. Penka, G. Grassi, O. I. Tolstikhin, J. Schneider, F. Jensen et al., Science 350, 790 (2015).
[13] A. Baltuška, T. Udem, M. Uiberacker, M. Hentschel, E. Goulielmakis, C. Gohle, R. Holzwarth, V. S. Yakovlev, A. Scrinzi, T. W. Hänsch, and F. Krausz, Nature (London) 421, 611 (2003).

[14] M. T. Hassan, T. T. Luu, A. Moulet, O. Raskazovskaya, P. Zhokhov, M. Garg, N. Karpowicz, A. M. Zheltikov, V. Pervak, F. Krausz, and E. Goulielmakis, Nature (London) 530, 66 (2016)

[15] E. Goulielmakis, M. Schultze, M. Hofstetter, V. S. Yakovlev, J. Gagnon, M. Uiberacker, A. L. Aquila, E. M. Gullikson, D. T. Attwood, R. Kienberger et al., Science 320, 1614 (2008).

[16] T. Gaumnitz, A. Jain, Y. Pertot, M. Huppert, I. Jordan, F. Ardana-Lamas, and H. J. Wörner, Opt. Express 25, 27506 (2017).

[17] E. Goulielmakis, Z.-H. Loh, A. Wirth, R. Santra, N. Rohringer, V. S. Yakovlev, S. Zherebtsov, T. Pfeifer, A. M. Azzeer, M. F. Kling et al., Nature (London) 466, 739 (2010).

[18] A. Wirth, M. T. Hassan, I. Grguraš, J. Gagnon, A. Moulet, T. T. Luu, S. Pabst, R. Santra, Z. A. Alahmed, A. M. Azzeer et al., Science 334, 195 (2011).

[19] S. Pabst, A. Sytcheva, A. Moulet, A. Wirth, E. Goulielmakis, and R. Santra, Phys. Rev. A 86, 063411 (2012).

[20] K. Gottfried and T.-M. Yan, Quantum Mechanics: Fundamentals (Springer, New York, 2003).

[21] C. Arnold, O. Vendrell, and R. Santra, Phys. Rev. A 95, 033425 (2017).

[22] S. Pabst, L. Greenman, P. J. Ho, D. A. Mazziotti, and R. Santra, Phys. Rev. Lett. 106, 053003 (2011).

[23] L. Greenman, P. J. Ho, S. Pabst, E. Kamarchik, D. A. Mazziotti, and R. Santra, Phys. Rev. A 82, 023406 (2010).

[24] T. Mazza, A. Karamatskou, M. Ilchen, S. Bakhtiarzadeh, A. J. 
Rafipoor, P. O'Keeffe, T. J. Kelly, N. Walsh, J. T. Costello et al., Nat. Commun. 6, 6799 (2015).

[25] S. Pabst and R. Santra, Phys. Rev. Lett. 111, 233005 (2013).

[26] C. Brif, R. Chakrabarti, and H. Rabitz, in Control of Quantum Phenomena, Advances in Chemical Physics, Vol. 148, edited by S. A. Rice and A. R. Dinner (Wiley, New York, 2012), pp. 1-76.

[27] S. J. Glaser, U. Boscain, T. Calarco, C. P. Koch, W. Köckenberger, R. Kosloff, I. Kuprov, B. Luy, S. Schirmer, T. Schulte-Herbrüggen et al., Europ. Phys. J. D 69, 279 (2015).

[28] M. Shapiro and P. Brumer, Principles of the Quantum Control of Molecular Processes (Wiley, New York, 2003).
[29] A. Karamatskou, S. Pabst, Y.-J. Chen, and R. Santra, Phys. Rev. A 89, 033415 (2014).

[30] A. Karamatskou, S. Pabst, Y.-J. Chen, and R. Santra, Phys. Rev. A 91, 069907(E) (2015).

[31] R. E. Goetz, A. Karamatskou, R. Santra, and C. P. Koch, Phys. Rev. A 93, 013413 (2016).

[32] R. E. Goetz, M. Merkel, A. Karamatskou, R. Santra, and C. P. Koch, Phys. Rev. A 94, 023420 (2016).

[33] M. Wollenhaupt, V. Engel, and T. Baumert, Annu. Rev. Phys. Chem. 56, 25 (2005).

[34] M. Amusia, L. Chernysheva, and V. Yarzhemsky, Handbook of Theoretical Atomic Physics (Springer, Heidelberg, 2012). 